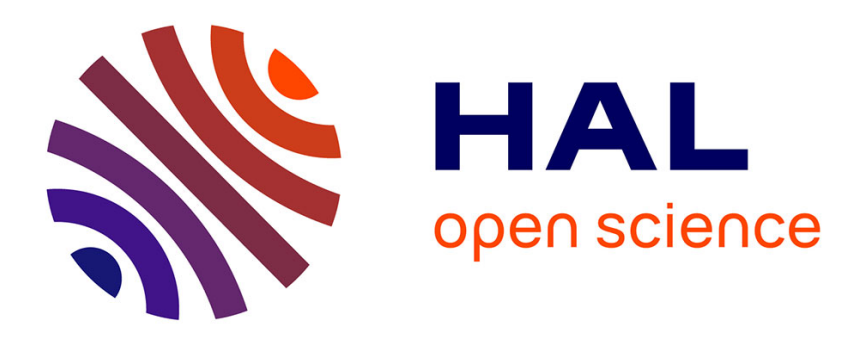

\title{
Les tailleresses de la Monnaie de Paris au travail (1640-1789) \\ Marion Delcamp
}

\section{To cite this version:}

Marion Delcamp. Les tailleresses de la Monnaie de Paris au travail (1640-1789). Revue Numismatique, 2015, 172e, pp.491-515. hal-01395984

\section{HAL Id: hal-01395984 \\ https://hal.science/hal-01395984}

Submitted on 15 Nov 2016

HAL is a multi-disciplinary open access archive for the deposit and dissemination of scientific research documents, whether they are published or not. The documents may come from teaching and research institutions in France or abroad, or from public or private research centers.
L'archive ouverte pluridisciplinaire HAL, est destinée au dépôt et à la diffusion de documents scientifiques de niveau recherche, publiés ou non, émanant des établissements d'enseignement et de recherche français ou étrangers, des laboratoires publics ou privés. 


\section{Marion DELCAMP*}

\section{Les tailleresses de la Monnaie de Paris au travail (1640-1789)}

Résumé - Aucune publication n'a jusqu'à présent eu pour objet les tailleresses des ateliers monétaires français, malgré l'extraordinaire exemple d'emploi manuel qualifié exercé par des femmes à l'époque moderne qu'elles constituent. L'exploitation des archives de la Monnaie de Paris pour la période 1640-1789 a permis d'exhumer de nombreuses sources qui permettent de mieux les cerner. Leur situation est relativement bien connue pour le Moyen Age, période pendant laquelle elles avaient une véritable place au sein du processus de production puisqu'elles étaient chargées de tailler les flans aux ciseaux avant leur impression par les monnayeurs. Elles faisaient à ce titre partie des « officiers de fabrication », corps constitué des monnayeurs, des ajusteurs et des tailleresses. Avec l'introduction et la généralisation à partir de 1640-1641 du laminoir, du balancier et surtout du coupoir réalisant désormais leur travail, elles furent affectées à la même tâche que les ajusteurs : limer les flans à l'aide d'écouennes. Le progrès technique a donc provoqué un doublon d'ouvriers pour exercer une même action, l'ajustage. La question du maintien des femmes au sein de l'atelier a assurément dû se poser. Bien que l'organisation médiévale n'ait plus eu de raison d'être en l'état, elle fut cependant maintenue au sein de l'atelier parisien et eut pour conséquence la marginalisation des tailleresses et la dévalorisation de leur travail. Plus tout à fait utiles, mal reconverties, difficilement limogeables, elles restèrent officiellement officiers des monnaies mais se virent peu à peu dépouillées de leurs droits.

Mots clés - Monnaie de Paris, tailleresse, ajustage, accueillement, apprentissage, conflits, productivité.

Summary - No publication has dealt so far with female workers of the French Mint (the tailleresses), despite the extraordinary example they represent of a physical qualified task performed by women during the Modern Era. A survey of the Paris Mint Monetary Archives between 1640 and 1789 revealed lists of female workers. Their situation is relatively well known for the Middle-Ages, during which they were responsible for cutting the blanks before striking. They were not more than a quarter to a fifth of the total workforce of fabricating officers. The introduction of the mill and later on of the screw-press, and especially that of the coupoir which was doing their previous job, led to assign them the same the same task as the adjusters'. The medieval organisation was no more justified but was maintained, resulting in their marginalisation and the gradual depreciation of their work. No more useful, unappropriately reconverted and difficult to dismiss, they remained officers of the Mint but were gradually deprived of their rights.

Keywords - Paris Mint, female workers of the Mint, filing, reception, apprenticeship, conflicts, productivity.

\footnotetext{
* Doctorante, école doctorale Cultures et Sociétés de l'Université Paris-Est/Marne-la-Vallée, laboratoire Analyse comparée des pouvoirs. marion.delcamp.ap@gmail.com
} 

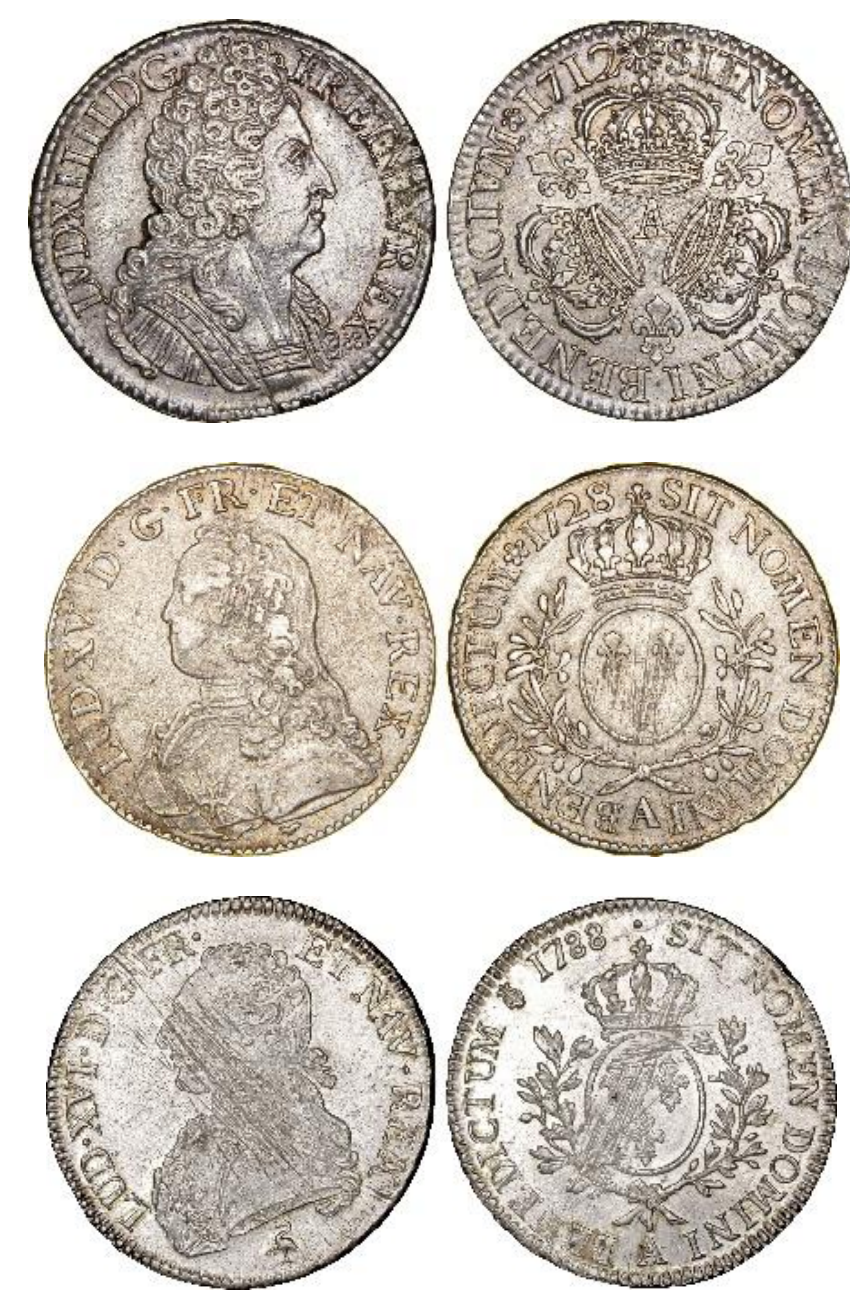

Figure 1. Ecus de Louis XIV ${ }^{1}$, Louis XV et Louis XVI portant des traces d'ajustage (OCGB.fr)

Les numismates savent reconnaître les traces d'ajustage que peuvent porter certaines des pièces de monnaies parvenues jusqu'à nous. Ces petites griffures (fig. 1) étaient l'œuvre des ajusteurs, présents dans toutes les Monnaies du royaume et chargés de retirer l'éventuel surplus de métal que pouvait contenir un flan sortant du coupoir. Ils n'étaient cependant pas les seuls à réaliser ce travail.

Dans son Traité des monnaies paru en 1764, Abot de Bazinghen précise à l'article «Monnoieur» que :

«[Les tailleresses] portent ce nom parce qu'avant la fabrication de la monnoie au moulin, chaque ajusteur avoit avec lui une tailleresse qui avec des cizoirs arrondissoit le flaon à mesure que l'ajusteur l'applatissoit avec le marteau; mais depuis l'établissement des moulins, les tailleresses font les mêmes opérations que les ajusteurs, qui sont obligés de leur donner à ajuster

\footnotetext{
1. Les monnaies de la série de l'écu d'argent de 60 sols, frappées au balancier après confection des flans au laminoir et au coupoir, ont été fabriquées exclusivement par Jean Warin, à Paris et à Lyon, de septembre 1641 à la mort de Louis XIII en mai 1643. On n'en a retrouvé aucune montrant des traces d'ajustage, Warin ayant obtenu de ses machines une perfection technique lui permettant de l'emporter sur Darmand Lorfelin (graveur général) et Louis de La Croix (maître parisien) avec lesquels il avait été mis en concurrence. Les premières stries d'ajustage apparaissaient sur des exemplaires frappés en province, à partir de 1646, dans le cadre de la généralisation de la fabrication mécanique.
} 
un quart dans chaque breve de chaque espece, de sorte que sur une breve de 100 marcs $^{2}$, les ajusteurs en travaillent 75 marcs, et les tailleresses $25 .^{3}{ }^{\star}$

Au Moyen Age et jusqu'au XVI ${ }^{\mathrm{e}}$ siècle ces femmes, qui ne devaient pas représenter plus d'un quart à un cinquième des effectifs de l'atelier, ajustaient donc les flans en coupant le surplus de métal à l'aide de gros ciseaux, alors qu'à partir du XVII ${ }^{\mathrm{e}}$ siècle elles n'agissent plus sur les flans qu'avec des limes, du fait de l'introduction des coupoirs permettant une production plus « standardisée ». Ce limage est d'ailleurs succinctement confirmé dans le Traité des monnaies de Boizard ${ }^{4}$, l'Encyclopédie de Diderot et d'Alembert ${ }^{5}$ ainsi que celle de Panckoucke ${ }^{6}$, par ailleurs monnayeur lillois ${ }^{7}$. Rappelons rapidement que le processus de fabrication monétaire après l'introduction du moulin et du balancier - la frappe au marteau étant définitivement interdite en 1645 - comportait plusieurs grandes étapes :

- Le métal, parvenu à la Monnaie sous forme de lingots, vaisselle, vieilles espèces, etc. était d'abord fondu en lingots ;

- Puis ceux-ci passaient au laminoir, ou moulin, afin d'en ressortir sous forme de lames, c'est-à-dire de plaques de métal très fines ;

- Des flans étaient ensuite découpés dans ces lames à l'aide d'un coupoir, sorte de machine à emporte-pièces, dont le diamètre était fonction de celui des espèces à fabriquer,

- Ces flans étaient ensuite ajustés par un ajusteur, longtemps appelé «ouvrier », ou une tailleresse, avec une lime appelée écouenne, c'est-à-dire mis au poids exigé par les ordonnances. Panckoucke fournit une bonne description de cette action précise :

«Le prévôt distribue ensuite les flans aux ouvriers et aux tailleresses pour les ajuster aux poids des espèces ; ils se servent de certains poids appelés déneraux pour les peser, et de limes en manière de râpes, avec des cannelures par angles entrans et sortans, appelées escouennes, pour limer les plus pesants jusqu'à ce qu'ils soient conformes aux déneraux, et ils rebutent ceux qui sont trop faibles. ${ }^{8}$ »

- La tranche des flans était marquée, à partir du milieu des années 1680, par la machine de Castaing $^{9}$;

- Enfin les flans étaient marqués ou «imprimés », par un monnayeur, au balancier.

L'ajusteur et la tailleresse avaient donc le même outil mais il semble que celle-ci ait davantage été perçue comme une « aide ajusteur » que comme une ouvrière à part entière, au vu de la petite quantité de matière qu'elle avait à travailler et de sa relative absence des sources archivistiques des ateliers monétaires. En conséquence, à l'inverse des groupes des monnayeurs et des ajusteurs, celui des tailleresses est méconnu. Pourtant, leur présence au sein des ateliers monétaires et leur place dans le processus de fabrication font d'elles un sujet d'étude original, complexe et riche d'enseignements. Elles constituent un rare exemple d'ouvrières qualifiées travaillant dans un secteur atypique, la petite métallurgie, de surcroît au service du roi. Le poste qu'elles occupaient était certes un emploi réservé puisque, théoriquement, seules les filles d'ajusteurs et de monnayeurs pouvaient y accéder ; il n'en demeure pas moins qu'il s'agissait pour l'époque d'un métier d'hommes réalisé par des femmes.

2. 1 marc $=244,75 \mathrm{~g}$.

3. ABOT DE BAZINGHEN 1764, p. 303.

4. BOIZARD 1692, p. 135-136.

5. DiDEROT et d'AlEMBERT 1772, t. 15 , p. 858.

6. PANCKOUCKE 1784-1788, t. 5, p. 142.

7. CLAIRAND 2014, p. 220.

8. PANCKOUCKE 1784-1788.

9. Cette machine était actionnée par un homme seulement qui pouvait marquer 20000 flans en une journée. Elle est l'invention du sieur Castaing, ingénieur du roi Louis XIV qui installa sa machine à la Monnaie de Paris en 1685 avant qu'elle soit envoyée dans toutes les Monnaies du royaume. BoIZARD 1714, p. 142. 
Peu de travaux scientifiques mentionnent les ouvrières des hôtels des monnaies et leur rôle dans le processus de fabrication. L'un des plus anciens ouvrages qui leur fait une petite place est celui d'Edouard Aubrée sur la Monnaie de Rennes, datant de 1903. Dans le cadre d'une étude généalogique, l'auteur aborde la situation générale des officiers de l'atelier breton et notamment celle des tailleresses, expliquant qu'au XVIII ${ }^{\mathrm{e}}$ siècle elles n'étaient plus reçues que pour reproduire le corps des officiers particuliers et n'étaient plus réellement employées à la fabrication de la monnaie ${ }^{10}$, sans justifier davantage ses propos. Un siècle plus tard, les actes du colloque de Nantes de 2003 donnent une courte définition générale de la tailleresse :

«Les tailleresses étaient chargées de la finition des flans (nettoyage, ébarbage...). L'Encyclopédie, qui consacre un chapitre à l'Art du monnayage, nous apprend que les tailleresses étaient « les femmes et les filles des monnayeurs ». Attention, il faut comprendre que les tailleresses étaient systématiquement recrutées parmi les femmes et les filles de monnayeurs, mais pas que toutes les femmes et les filles de monnayeurs devenaient obligatoirement tailleresses. ${ }^{11}$ »

En pratique, il est possible de trouver, pour l'atelier parisien, des cas de grâces royales permettant à une femme de se faire accueillir à la Monnaie sans avoir eu de père monnayeur ou ajusteur $^{12}$. D'autre part, contrairement à ce que l'article de l'Encyclopédie de Diderot et d'Alembert pourrait laisser penser, nous n'avons trouvé aucune trace de femme demandant à se faire recevoir à la Monnaie de Paris en faisant valoir son statut d'épouse de monnayeur ou d'ajusteur. En revanche, une étude prosopographique des officiers de fabrication de la Monnaie de Paris ${ }^{13}$ met en lumière quelques cas de mariages endogamiques, voire homogamiques, mais sans que l'on sache s'ils ont été célébrés avant ou après l'incorporation des tailleresses concernées ${ }^{14}$. Très récemment, la grande étude de Jérôme Jambu sur les ateliers monétaires de Caen et Saint-Lô a par exemple consacré plusieurs lignes à ces femmes officiers et à leur rôle au sein des Monnaies ${ }^{15}$. Évoquant leur présence au sein de l'atelier de Saint-Lô, il explique qu'elles étaient les «mères ou filles [des ouvriers de la Monnaie] » travaillant avec les ajusteurs. Il n'existe par ailleurs aucune étude sur le personnel de la Monnaie de Paris en particulier, pourtant première Monnaie du royaume en termes d'ancienneté et de production. Seul Jean-Marie Darnis a étudié l'atelier parisien entre 1795 à $1826^{16}$. Cependant, l'administration de la Monnaie et les progrès techniques successivement mis en place en son sein sont les objets principaux de cette étude ; les personnels d'exécution y sont très peu évoqués. L'étude d'une frange minoritaire du personnel de l'hôtel des monnaies parisien, dont on a des traces matérielles du travail réalisé sur les espèces conservées, est donc une première approche dans une perspective d'étude globale de ce corps de travailleurs ${ }^{17}$.

\section{Combien de tailleresses ?}

10. AUBREE 1903, p. 43.

11. SALAÜN 2003, p. 13.

12. Comme par exemple Marie Cécile et Marie Claude Langlois, reçues à la Monnaie le 3 juillet 1690 (AN, $\mathrm{Z}^{1 \mathrm{~B}}$ 572), Marie De La Haye reçue le 15 mars 1691 (AN, $Z^{1 \mathrm{~B}}$ 573) ou encore Marie Jacqueline et Pétronille Françoise Le Vieux, reçues le 28 septembre 1718 (AN, $Z^{1 \mathrm{~B}} 577$ ), toutes munies de lettres de relief et d'interruption. 13. Ce travail a été mené pour l'effectif des tailleresses de la Monnaie de Paris sur la période 1645-1789 dans le cadre de nos deux années de master.

14. Comme Marie Jeanne Boudin, mariée à un certain Couteux dont la profession n'est pas précisée mais dont le nom de famille est celui d'une famille présente à la Monnaie au XVIII ${ }^{\mathrm{e}}$ siècle (AN, $\mathrm{Z}^{\mathrm{B}}$ 586) ou Geneviève Auboust qui s'est mariée à Simon Bazard, ajusteur (AN, $\left.\mathrm{Z}^{1 \mathrm{~B}} 572\right)$.

15. JAMBU 2013, p. 71.

16. DARNIS 1988.

17. L'étude de l'intégralité du corps des travailleurs de l'atelier monétaire de Paris entre 1550 et 1790 est le sujet de notre thèse de doctorat actuellement en cours de réalisation. 
Les sources concernant les femmes «officiers ${ }^{18} »$ de l'hôtel des monnaies de Paris proviennent de deux fonds principaux conservés aux Archives Nationales, les archives de la Cour des monnaies de Paris ${ }^{19}$ et les archives de la Compagnie des monnayeurs de Paris ${ }^{20}$, ainsi que des archives de la Monnaie de Paris, conservées quant à elles au Centre des Archives Économiques et Financières (CAEF, Savigny-le-Temple). Les premiers résultats que l'on en a tirés sont les effectifs des tailleresses reçues au sein de l'atelier parisien depuis l'introduction du moulin dans la fabrication des espèces ${ }^{21}$. Ils permettent de mettre en évidence les vagues de réceptions, toujours corrélées avec les périodes de fortes productions monétaires :

\begin{tabular}{|c|c|c|c|c|c|}
\hline $1648: 1$ & $1673: 1$ & $1706: 2$ & $1723: 4$ & $1759: 1$ & $1786: 3$ \\
\hline $\mathbf{1 6 5 1}: \mathbf{8}$ & $1679: 4$ & $1707: 3$ & $\mathbf{1 7 2 6}: \mathbf{6}$ & $1764: 1$ & $1787: 3$ \\
\hline $\mathbf{1 6 5 2}: \mathbf{4}$ & $1685: 2$ & $\mathbf{1 7 0 9}: \mathbf{1 4}$ & $1729: 1$ & $1769: 1$ & $1788: 1$ \\
\hline $\mathbf{1 6 5 3}: \mathbf{6}$ & $1687: 1$ & $1710: 2$ & $1733: 2$ & $1773: 1$ & $1789: 3$ \\
\hline $1654: 1$ & $\mathbf{1 6 9 0}: \mathbf{1 8}$ & $1713: 2$ & $1737: 1$ & $1774: 4$ & \\
\hline $1655: 1$ & $1691: 1$ & $1716: 3$ & $1744: 2$ & $1775: 1$ & \\
\hline $1656: 2$ & $1703: 1$ & $\mathbf{1 7 1 8}: \mathbf{6}$ & $1754: 1$ & $1783: 6$ & \\
\hline $1662: 1$ & $1704: 4$ & $1720: 4$ & $\mathbf{1 7 5 5}: \mathbf{6}$ & $1784: 4$ & \\
\hline $1667: 1$ & $1705: 1$ & $1721: 1$ & $\mathbf{1 7 5 6}: \mathbf{6}$ & $\mathbf{1 7 8 5}: \mathbf{6}$ & \\
\hline
\end{tabular}

Figure 2. Nombre des tailleresses ayant intégré la Monnaie de Paris entre 1648 et 1789

- 1651-1653 : arrivée massive d'argent hispano-américain devant être refondu ;

- 1690 : fonte des vaisselles d'argent du roi et des particuliers et début des réformations louis-quatorziennes décidées en 1689 ;

- 1709 : refonte générale des anciennes espèces et fabrication de nouvelles espèces ;

- 1718 : réforme monétaire qui créé l'écu d'argent dit « de France et de Navarre ».

- 1726 : refonte générale qui marque le début de la stabilité monétaire ;

- 1755-1756 : au cœur de la guerre de Sept Ans, augmentation de la frappe de pièces d'or pour solder les troupes et financer les alliances ${ }^{22}$;

- 1785: réforme du louis d'or afin de l'établir sur le pied des autres monnaies européennes $^{23}$.

Ces chiffres montrent clairement que, lors des accroissements d'activité, l'atelier mobilisait en nombre des officiers de fabrication féminins pour répondre à la demande royale, ce qui ne laisse planer aucun doute quant à leur présence et à leur utilité effectives au sein de l'atelier jusqu'à la fin de la période moderne. La fonte des vaisselles et la première réformation du règne de Louis XIV, en 1689-1690, constitue à ce titre un record, avec 18 nouvelles tailleresses accueillies en un an.

La procédure initiale d'intégration à l'établissement se déroulait de la même façon que pour un homme ${ }^{24}$ et, contrairement à ce qui pouvait exister dans d'autres ateliers français, celui de

18. Le corps des « officiers de fabrication » était composé des monnayeurs, des ajusteurs et des tailleresses.

19. AN, sous-série $\mathrm{Z}^{1 \mathrm{~B}}$.

20. AN, sous-série T 1491.

21. Nous ne disposons malheureusement pas des effectifs antérieurs à 1648 .

22. ClaIRAND 1996. On peut penser que la refonte des vaisselles d'argent de 1759 n'a pas demandé de réceptions supplémentaires du fait des 12 précédemment réalisées en 1755-1756.

23. THUILLIER 2005.

24. La différence entre l'intégration d'un homme et d'une femme arrivant plus loin dans le cursus d'un(e) nouvel(le) accueilli(e). 
Paris n'imposait pas d'âge minimum, seulement un âge maximum théorique de 25 ans $^{25}$. La procédure se décomposait en quatre temps : la demanderesse présentait d'abord sa requête à la Cour des monnaies qui ordonnait qu'elle soit transmise aux prévôts et lieutenants des monnayeurs, des ajusteurs et des tailleresses de la Monnaie concernée. Si les prévôts et lieutenants donnaient leur consentement, le procureur général du roi ordonnait une information de vie et mœurs de la postulante, qu'il transmettait à la Cour. Si la Cour des monnaies considérait cette information bonne et valable, elle la validait et la renvoyait au procureur général. Pour terminer, la Cour renvoyait la demanderesse devant son prévôt et son lieutenant pour être « accueillie à son droit».

\section{La réception d'une nouvelle tailleresse}

Bien qu'utiles à l'atelier, Abot de Bazinghen notait que les tailleresses n'avaient cependant pas d'apprentissage, puisqu'elles étaient «simplement reçues ${ }^{26}$ », alors que les monnayeurs et les ajusteurs étaient «accueillis », réalisaient leur «épreuve» puis étaient «reçus. ${ }^{27}$ » D'ailleurs, les droits à régler lors des accueillement, épreuve et réception des années $1690^{28}$, $1756^{29}$ et $1767^{30}$ ne mentionnent effectivement que la réception pour les tailleresses. Ci-dessous figurent les résumés des édits pris à ce sujet par la Cour des monnaies ne concernant que les officiers de fabrication de l'atelier monétaire de Paris, suivi du détail plus particulier des droits de réception d'une tailleresse.

À la suite de l'édit de 1690, une modification est réalisée par la Compagnie des monnayeurs, ajusteurs et tailleresses, daté du 6 février 1691. Elle stipule qu'à partir de ce jour, les bouquets remis le jour de l'épreuve des recuiteurs et des ricochons ${ }^{31}$ et celui de la prestation de serment à la Cour des monnaies seraient remplacés par 8 livres de bougies que le récipiendaire déposerait au greffe de la Cour et seraient distribuées entre ses membres et les officiers de la Compagnie des monnayeurs, ajusteurs et tailleresses. De plus, le repas dû aux travailleurs de la Monnaie au retour de la Cour, après la prestation de serment, est également supprimé. Il est remplacé par le don d'un jeton d'argent de 2 gros $^{32}$ à chaque ouvrier et chaque monnayeur en poste ( aux actuels »). Cela modifie sensiblement le coût d'un accueillement pour un nouveau monnayeur et un nouvel ajusteur, mais pas pour une tailleresse puisque déjà, en 1690, les femmes n'avaient à offrir aucune denrée alimentaire en prévision du banquet. Cette pratique est certainement héritée de la bienséance du Moyen Age qui excluait les femmes de ce genre de manifestations « masculines. »

25. Il est facile de trouver des officiers relevés par grâce royale, afin qu'ils puissent se faire accueillir à l'atelier, malgré leur âge «avancé », dans la sous-série $Z^{1 \mathrm{~B}}$ des Archives Nationales conservant les « lettres de provisions d'offices » pour les Monnaies du royaume (cotes $Z^{1 \mathrm{~B}} 448$ à 600 ).

26. Là se trouve la différence sexuée. ABOT DE BAZINGHEN 1764, p. 307.

27. Accueillement, épreuve, réception sont les trois temps de l'incorporation d'un monnayeur et d'un ajusteur à la Monnaie.

28. AN, T $1491^{12}$ (registre). C'est le plus ancien texte réglant les frais d'accueillement retrouvé.

29. Arrest de la Cour des monnoyes du vingt-un juillet 1756 portant homologation de l'état et règlement des droits que les monnoyeurs, ajusteurs, tailleresses, accueillis, ajusteurs et monnoyeurs de la Monnoye de Paris, du titre et serment de France, doivent payer lors de leurs accueillemens, épreuves et réceptions en ladite Monnoye, Paris, 21 juillet 1756. Paris, G. LAMESLE, 1756.

35. Arrêt de la Cour des monnoies du 23 mars 1767 portant homologation de l'état et règlement des droits que les monnoyeurs, ajusteurs et tailleresses, accueillis, ajusteurs et monnoyeurs de la Monnoie de Paris, du titre et serment de France, doivent payer lors de leurs accueillemens, épreuves et réceptions en ladite Monnoie, Paris, 23 mars 1767. Paris, imprimerie royale, 1767.

31. C'est-à-dire les apprentis ajusteurs et monnayeurs, ce qui confirme qu'en 1690 les tailleresses n'avaient pas d'épreuve.

32. Soit environ $7,64 \mathrm{~g}$. 


\begin{tabular}{|c|c|c|c|c|c|c|}
\hline \multicolumn{7}{|c|}{ Droits à régler en 1690 (effectifs 1691) } \\
\hline & \multicolumn{2}{|c|}{ MONNAYEUR (29) } & \multicolumn{2}{|c|}{ AJUSTEUR (23) } & \multicolumn{2}{|c|}{ TAILLERESSE (31) } \\
\hline & Quantité & Valeur & Quantité & Valeur & Quantité & Valeur \\
\hline \multicolumn{7}{|c|}{ ACCUEILLEMENT } \\
\hline ARGENT & & $28 \mathrm{~L} 8 \mathrm{~s}$ & & $28 \mathrm{~L} 8 \mathrm{~s}$ & \multirow{2}{*}{\multicolumn{2}{|c|}{ Aucune mention }} \\
\hline JETONS de 2 gros & 68 & $60 \mathrm{~L} 12 \mathrm{~s} 8 \mathrm{~d}$ & 68 & $60 \mathrm{~L} 12 \mathrm{~s} 8 \mathrm{~d}$ & & \\
\hline \multicolumn{7}{|c|}{ EPREUVE } \\
\hline \multicolumn{7}{|c|}{ Faire donner une messe le jour de l'épreuve en la chapelle de l'Hôtel de la monnaie } \\
\hline ARGENT & & $174 \mathrm{~L} 16 \mathrm{~s}$ & & $160 \mathrm{~L} 16 \mathrm{~s}$ & \multirow{9}{*}{\multicolumn{2}{|c|}{ Aucune mention }} \\
\hline JETONS de 2 gros & 201 & $179 \mathrm{~L} 4 \mathrm{~s} 6 \mathrm{~d}$ & 199 & $177 \mathrm{~L} 8 \mathrm{~s} 10 \mathrm{~d}$ & & \\
\hline BOUGIES (12 à la livre) & 136 & $?$ & 134 & $?$ & & \\
\hline \begin{tabular}{|l|} 
Boîtes d'ANIS \\
\end{tabular} & 11 & $?$ & 10 & $?$ & & \\
\hline Bouteilles de VIN & 10 & $?$ & 10 & $?$ & & \\
\hline Plats de VIANDES & 3 & $?$ & 3 & $?$ & & \\
\hline PAINS & 36 & $?$ & 36 & $?$ & & \\
\hline PAINS BENIS & 4 & $?$ & 4 & $?$ & & \\
\hline BOUQUETS & 66 & $?$ & 66 & $?$ & & \\
\hline \multicolumn{7}{|c|}{ RECEPTION } \\
\hline ARGENT & \multirow{3}{*}{\multicolumn{2}{|c|}{ Aucune mention }} & \multirow{3}{*}{\multicolumn{2}{|c|}{ Aucune mention }} & & $100 \mathrm{~L}$ \\
\hline \begin{tabular}{|l|} 
JETONS d'1,5 gros \\
\end{tabular} & & & & & 62 & $41 \mathrm{~L} 6 \mathrm{~s} 8 \mathrm{~d}$ \\
\hline \begin{tabular}{|l|} 
JETONS de 2 gros \\
\end{tabular} & & & & & 64 & $57 \mathrm{~L} 1 \mathrm{~s} 4 \mathrm{~d}$ \\
\hline \begin{tabular}{|c|} 
TOTAL d'une \\
incorporation en 1690
\end{tabular} & \multicolumn{2}{|c|}{$\begin{array}{l}443 \text { L } 1 \text { s } 2 \text { d + } 136 \text { bougies + } \\
\text { denrées alimentaires }\end{array}$} & \multicolumn{2}{|c|}{$\begin{array}{l}427 \text { L } 5 \text { s } 6 \text { d + } 134 \text { bougies + } \\
\text { denrées alimentaires }\end{array}$} & \multicolumn{2}{|c|}{198 L $8 \mathrm{~s}$} \\
\hline
\end{tabular}

Figure 3. Les droits fixés par l'arrêt de la Cour des monnaies du 25 novembre 1690.

Détail des frais de réception d'une tailleresse en 1690 (inchangés en février 1691) :

* 100 1. à donner au prévôt des ouvriers ou à son lieutenant, qui les distribuera comme suit :

- 44 1. au receveur de la communauté pour le droit de boîte

- 24 1. au prévôt des monnayeurs

- 40 s. au clerc des monnayeurs pour la semonce ${ }^{33}$

- $40 \mathrm{~s}$. au clerc des ajusteurs pour la semonce

- Le prévôt ou son lieutenant gardera 24 1. entre ses mains ainsi que les 4 1. du droit d'assiette

Les 24 1. remises au prévôt des monnayeurs et les 28 1. gardées par le prévôt des ouvriers seront partagées entre les travailleurs déjà en poste comme bénécés ${ }^{34}$.

* Des jetons d'argent de 2 gros chacun à remettre au receveur, distribués comme suit :

- 2 par officier

- 1 par monnayeur et 1 par ajusteur

* 2 jetons d'argent d' 1 gros et demi ${ }^{35}$ chacun par tailleresse déjà en poste.

Ces jetons remplacent la collation ${ }^{36}$ et la bienvenue ainsi que le droit de siège ${ }^{37}$.

En cas d'absence de certains officiers, leurs jetons seront placés dans la caisse commune de la Compagnie, suivant la délibération de la communauté du 9 mai $1679^{38}$.

33. C'est-à-dire l'annonce d'un nouvel accueilli, faite par les clercs à la communauté.

34. Les bénécés désignent les sommes reçues par les officiers de fabrication de la Monnaie en poste (les 6 officiers ainsi que le reste des travailleurs) lors de l'accueillement d'un nouveau monnayeur ou d'un nouvel ajusteur (donc pas au moment de l'épreuve de celui-ci) et lors de la réception d'une tailleresse. En théorie, ces sommes, confiées par le récipiendaire à son prévôt, doivent être redistribuées «équitablement » aux travailleurs de l'atelier.

35. Soit environ $5,73 \mathrm{~g}$.

36. C'est-à-dire le banquet. Ce qui confirme qu'elles n'y étaient pas conviées.

37. Les droits de boîte, d'assiette et de siège étaient des taxes supportées par les nouveaux reçus qui disparaissent au XVIII ${ }^{\mathrm{e}}$ siècle dont nous n'avons pas trouvé de définition exacte.

38. Cette information contenue dans l'arrêt de la Cour des monnaies de 1690 demeure valable lors des arrêts futurs de 1756 et 1767 . 
Le rôle de personnel dont nous disposons pour l'année 1690 recense 31 tailleresses à la Monnaie de Paris. Chaque nouvelle tailleresse reçue à partir du 25 novembre 1690 jusqu'au 6 février 1691 devait donc faire «frapper » 62 jetons d'1,5 gros (fig. 4) ${ }^{39}$. En ce qui concerne la valeur jetons d'argent, on a pu l'estimer grâce aux tarifs des matières d'or et d'argent des années $1690^{40}, 1759^{41}$ et $1771^{42}$, afin d'avoir une idée plus précise des coûts qu'ils engendraient (fig. $5)$.
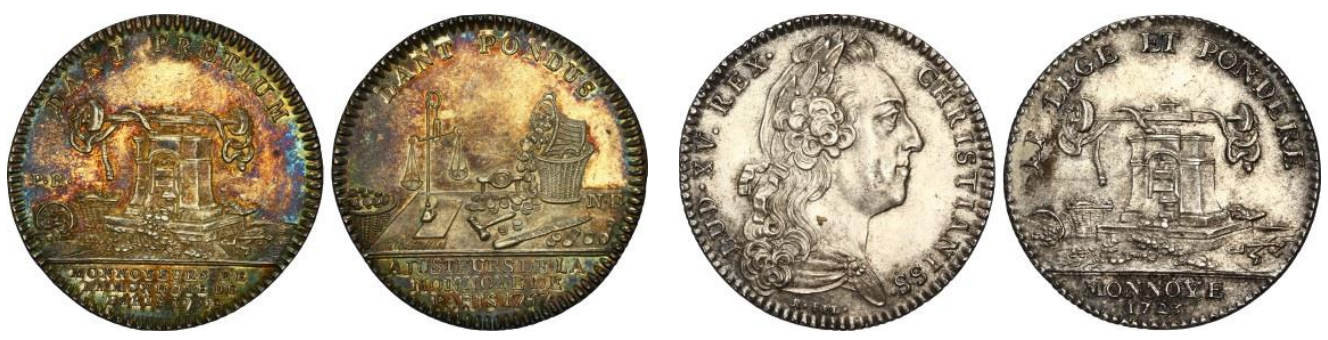

«Ils donnent la valeur - Ils donnent le poids » pour celui de gauche. «Par la loi et par le poids » pour celui de droite, c'est-à-dire «par le titre et par le poids » car, à l'époque moderne, la « loi »signifiait le «titre » de la monnaie.

Figure 4. Deux jetons frappés par les ajusteurs de la Monnaie de Paris (CCGB.fr)

\begin{tabular}{|l|c|c|c|}
\hline & $\mathbf{1 6 9 0}$ & $\mathbf{1 7 5 9}$ & $\mathbf{1 7 7 1}$ \\
\hline $\mathbf{1}$ marc d'argent & $281.10 \mathrm{~s} 10 \mathrm{~d}$ & $541.7 \mathrm{~s}$ & $491.1 \mathrm{~d}$ \\
\hline $\mathbf{4}$ onces & $121.9 \mathrm{~s} 4 \mathrm{~d}$ & $271.3 \mathrm{~s} 6 \mathrm{~d}$ & $241.10 \mathrm{~s}$ \\
\hline $\mathbf{2}$ onces & $61.4 \mathrm{~s} 8 \mathrm{~d}$ & $131.11 \mathrm{~s} 9 \mathrm{~d}$ & $121.5 \mathrm{~s}$ \\
\hline $\mathbf{1}$ once & $31.2 \mathrm{~s} 4 \mathrm{~d}$ & $61.15 \mathrm{~s} 10 \mathrm{~d}$ & $61.2 \mathrm{~s} 6 \mathrm{~d}$ \\
\hline $\mathbf{1}$ gros & $8 \mathrm{~s} 7 \mathrm{~d}$ & $16 \mathrm{~s} 11 \mathrm{~d}$ & $15 \mathrm{~s} 3 \mathrm{~d}$ \\
\hline $\mathbf{2}$ gros & $17 \mathrm{~s} 2 \mathrm{~d}$ & $11.13 \mathrm{~s} 11 \mathrm{~d}$ & $11.10 \mathrm{~s} 7 \mathrm{~d}$ \\
\hline
\end{tabular}

Figure 4. Tableau récapitulatif du cours de l'argent et/ou des jetons d'argent en 1690, 1759 et 1771

Les biens matériels et alimentaires à fournir méritent également quelques explications. La nature des bougies n'est pas précisée, l'arrêt ne précisant que leur poids « de 12 à la livre. ${ }^{43}$ » Sous l'Ancien Régime, la noblesse française et le clergé s'éclairaient avec des cierges en cire d'abeille et laissaient au peuple l'éclairage au suif ${ }^{44}$. Il aurait été intéressant de disposer de cette information car le prix des bougies de cire d'abeille (plus performantes, elles éclairaient mieux,

39. Attention cependant à ce terme, qui est celui utilisé par la Cour des monnaies. Il n'est pas certain que les nouveaux venus aient effectivement eu à faire frapper eux-mêmes, à chaque fois, de nouveaux jetons : sans doute pouvaient-ils en racheter de plus anciens, déjà fabriqués : nous y reviendrons. Ces jetons feront l'objet d'un prochain article qui présentera leur forme et les cadres de leur utilisation en préparation.

40. Évaluation et tarif des espèces, vaisselles et matières d'or et d'argent, Paris, 12 janvier 1690. Paris, F. Léonard, 1690. Pour ce texte on dispose du prix auquel les jetons de France sont rachetés ; c'est celui-ci qui est indiqué dans le tableau.

41. Évaluation et tarif du prix que le roi veut et ordonne être payé aux hôtels des monnoies, des matières, vaisselles et ouvrages d'or et d'argent qui y seront apportées », Paris, 5 novembre 1759. Paris, imprimerie royale, 1759.

42. Arrest du Conseil d'État du Roi, et lettres patentes sur icelui, du 15 Septembre 1771. Qui fixent le prix auquel les matières d'Or \& d'Argent, seront reçues au Change des hôtel des Monnoies, Paris, 15 septembre 1771. Paris, P.-G. Simon, 1771.

43. C'est-à-dire que l'on fabriquait 12 bougies dans une livre de cire ou de suif, donc chacune pesait $40 \mathrm{~g}$. 44. La bougie était faite en cire d'abeille et la chandelle en suif. Mais il pouvait y avoir à l'époque confusion entre les deux termes. 
brûlaient plus lentement et ne sentaient pas mauvais) était bien plus élevé que celui des bougies en suif (graisse animale), ce qui ferait augmenter de beaucoup le total des droits à régler.

La présence des boîtes d'anis ${ }^{45}$, quant à elles, peut surprendre. Pourtant, cette épice est l'une des plus anciennes et des plus utilisées par l'homme, dont on a trouvé la trace dans l'Égypte ancienne. Les Egyptiens l'utilisaient en pharmacie, pour fabriquer des onguents ou des élixirs. À l'époque moderne, elle était très appréciée en bonbons, comme la réglisse ou le clou de girofle, pour améliorer une haleine rarement délicate, et souvent utilisée en cuisine. Il n'est donc pas étonnant que l'anis fasse partie des denrées à offrir pour le banquet d'accueillement, mais également comme simple «don de courtoisie».

Le vin, les plats de viandes et les pains servent quant à eux au banquet d'après serment. À ce propos, il faut faire remarquer que les tailleresses n'avaient aucun plat de ce genre à offrir pour les cérémonies. Il semble donc que leur seule réception les privait de banquet; à moins, bien-sûr, que les monnayeurs et les ajusteurs nouvellement incorporés le leur offrissent par galanterie, ce dont nous doutons. Les plats de viandes étaient composés de différentes volailles : poulets d'Inde - c'est-à-dire des dindes ou dindons, poulets des Indes occidentales ${ }^{46}$-, chapons $^{47}$ et poulets ${ }^{48}$ - deux chapons valant apparemment trois poulets si l'on en croit les instructions de l'arrêt. Le vin ${ }^{49}$ quant à lui était l'élément indissociable des festivités de l'époque moderne. Il était bien évidemment empreint de religion tout comme l'était la communauté des monnayeurs, ajusteurs et tailleresses de la Monnaie de Paris ${ }^{50}$ et le met suivant. Le pain faisait partie intégrante de l'alimentation quotidienne depuis le Moyen Age, il n'est donc pas étonnant de le compter parmi les denrées alimentaires consommées pendant un banquet. Les pains bénis servaient par contre à la messe qui avait lieu en la chapelle de l'hôtel de la Monnaie le jour de l'épreuve. Les bouquets étaient de fleurs, mais nous n'avons aucune précision à ce propos.

L'avenant de 1691 met cependant fin à ces pratiques festives tant les abus étaient courants : de nombreux «maîtres » profitaient des largesses des nouveaux accueillis qui n'osaient refuser d'offrir ce qu'ils leurs demandaient. Ce texte n'est cependant qu'une très mince modification des frais supportés par les nouveaux arrivants, la première évolution importante datant de 1756.

45. Nous n'avons pas trouvé le prix de l'anis pour cette période.

46. D'après D'AVENEL 1894-1926, t. 4 p. 290, un poulet d'Inde était vendu $11.10 \mathrm{~s}$ en région parisienne (à Brétigny-sur-Orge) en 1668 et un « gros dindon » était vendu 11.15 s à Orléans en 1690.

47. Nous n'avons pas trouvé de prix fixes et cohérents pour les chapons. Par exemple, un «chapon de Bruges » était vendu 75 s en 1697 ( «en France »), alors qu'un « chapon gras » était vendu 66 s et un simple chapon 36 s. D’AVENEL 1894-1926, t. 4, p. 271-273.

48. Un poulet était vendu $8 \mathrm{~s}$ en moyenne à Paris au début du XVIII siècle. D’AVENEL 1894-1926, t. 4 p. $271-273$. 49. La bouteille de vin $(93 \mathrm{cl})$ était vendue $10 \mathrm{~s}$ à Paris dans les premières années du XVIII' ${ }^{\mathrm{e}}$ siècle. D'AVENEL 18941926, t. 4 p. 181.

50. La communauté avait entre autres un saint patron (Saint Eloi), une charité (la Maladrerie du Roule) et le serment qu'elle faisait prêter aux nouveaux accueillis contenait quelques citations de la Bible, comme cela se faisait beaucoup au sein des compagnies d'ouvriers qualifiés (tailleurs de pierre ou orfèvres). Cette dimension est développée dans DELCAMP 2014, p. 66-67 et 83-85. 


\begin{tabular}{|c|c|c|c|c|c|c|}
\hline \multicolumn{7}{|c|}{ Droits à régler en 1756 (effectifs 1754) } \\
\hline & \multicolumn{2}{|c|}{ MONNAYEUR (23) } & \multicolumn{2}{|c|}{ AJUSTEUR (23) } & \multicolumn{2}{|c|}{ TAILLERESSE (31) } \\
\hline & Quantité & Valeur & Quantité & Valeur & Quantité & Valeur \\
\hline \multicolumn{7}{|c|}{ ACCUEILLEMENT } \\
\hline \begin{tabular}{|l|} 
ARGENT \\
\end{tabular} & & $79 \mathrm{~L} 10 \mathrm{~s}$ & & $79 \mathrm{~L} 10 \mathrm{~s}$ & \multirow{2}{*}{\multicolumn{2}{|c|}{ Aucune mention }} \\
\hline \begin{tabular}{|l|} 
JETONS de 2 gros \\
\end{tabular} & 81 & $137 \mathrm{~L} 7 \mathrm{~s} 3 \mathrm{~d}$ & 58 & $98 \mathrm{~L} 7 \mathrm{~s} 2 \mathrm{~d}$ & & \\
\hline \multicolumn{7}{|c|}{ EPREUVE } \\
\hline ARGENT & & $184 \mathrm{~L} 10 \mathrm{~s}$ & & $184 \mathrm{~L} 10 \mathrm{~s}$ & \multirow{6}{*}{\multicolumn{2}{|c|}{ Aucune mention }} \\
\hline BOUQUETS & & $6 \mathrm{~L}$ & & $6 \mathrm{~L}$ & & \\
\hline \begin{tabular}{|l|} 
JETONS de 2 gros \\
\end{tabular} & 391 & $663 \mathrm{~L} 1 \mathrm{~s} 5 \mathrm{~d}$ & 267 & $452 \mathrm{~L} 15 \mathrm{~s} 9 \mathrm{~d}$ & & \\
\hline BOUGIES (8 à la livre) & 15 livres $(120 \mathrm{~b})$. & $?$ & 15 livres $(120$ b. $)$ & ? & & \\
\hline BOUGIES (12 à la livre) & $171+x$ & $?$ & $171+x$ & $?$ & & \\
\hline \begin{tabular}{|l|} 
LOUIS D'OR en argent \\
\end{tabular} & & $16 \mathrm{~L}$ & & $16 \mathrm{~L}$ & & \\
\hline \multicolumn{7}{|c|}{ RECEPTION } \\
\hline ARGENT & \multirow{3}{*}{\multicolumn{2}{|c|}{ Aucune mention }} & \multirow{3}{*}{\multicolumn{2}{|c|}{ Aucune mention }} & & $143 \mathrm{~L} 10 \mathrm{~s}$ \\
\hline JETONS d'1,5 gros & & & & & 62 & $78 \mathrm{~L} 10 \mathrm{~s} 8 \mathrm{~d}$ \\
\hline \begin{tabular}{|l|} 
JETONS de 2 gros \\
\end{tabular} & & & & & 58 & $98 \mathrm{~L} 7 \mathrm{~s} 2 \mathrm{~d}$ \\
\hline \begin{tabular}{|c|} 
TOTAL d'une \\
incorporation en 1756 \\
\end{tabular} & \multicolumn{2}{|c|}{$\begin{array}{c}1086 \text { L } 8 \text { s } 8 \text { d + 291+x bougies } \\
\text { (des } 8 \text { et } 12 \text { à la livre) }\end{array}$} & \multicolumn{2}{|c|}{\begin{tabular}{|c|}
837 L 2 s 11 d + 291+x bougies \\
(des 8 et 12 à la livre) \\
\end{tabular}} & \multicolumn{2}{|c|}{320 L 7 s $10 d$} \\
\hline
\end{tabular}

Figure 5. Les droits fixés par l'arrêt de la Cour des monnaies du 21 juillet 1756

Détails des frais de réception d'une tailleresse en 1756 :

* 100 1. à donner au prévôt des ajusteurs et tailleresses qui les distribuera comme suit :

- 44 1. à remettre au syndic-receveur pour le droit de boîte.

- 28 1. partagées entre les ajusteurs qui ont droit aux bénécés.

- 24 1. au prévôt des monnayeurs qui les distribuera aux monnayeurs ayant droit aux bénécés.

- 4 1. pour les 2 clercs de la Compagnie pour leur droit de semonce ( 2 fois $40 \mathrm{~s}$ ).

* 42 1. pour l'arrêt de la Cour qui renvoie la tailleresse par devant les prévôts et lieutenants.

* 11.10 s pour la lettre de réception, dite «Petite Lettre. ${ }^{51}$ »

* Des jetons d'argent de 2 gros chacun frappés sur les carrés de la Compagnie et non d'autres, distribués comme suit :

- $2 /$ officier

- $1 /$ monnayeur et 1/ajusteur

* 2 jetons d'argent d'1 gros et demi chacun par tailleresse, frappés sur les carrés de la Compagnie et non d'autres.

* Aucun repas supplémentaire à ces frais.

51. Ces petites lettres n'étaient pas des lettres de provision d'office comme on a coutume d'en trouver pour les autres officiers des ateliers monétaires. Nous n'en avons à ce jour retrouvé aucune pour Paris. Les monnayeurs, les ajusteurs et les tailleresses faisaient partie de ces franges de petits officiers au statut flou, organisés telle une corporation mais prenant néanmoins leurs fonctions par arrêt de la Cour des monnaies de Paris, formalité à haute valeur juridique. 


\begin{tabular}{|c|c|c|c|c|c|c|}
\hline \multicolumn{7}{|c|}{ Droits à régler en 1767 (effectifs 1783) } \\
\hline & \multicolumn{2}{|c|}{ MONNAYEUR (26) } & \multicolumn{2}{|c|}{ AJUSTEUR (25) } & \multicolumn{2}{|c|}{ TAILLERESSE (22) } \\
\hline & Quantité & Valeur & Quantité & Valeur & Quantité & Valeur \\
\hline \multicolumn{7}{|c|}{ ACCUEILLEMENT } \\
\hline \begin{tabular}{|l|} 
ARGENT \\
\end{tabular} & & $121 \mathrm{~L}$ & & $121 \mathrm{~L}$ & \multirow{2}{*}{\multicolumn{2}{|c|}{ Aucune mention }} \\
\hline JETONS de 24 au marc & 95 & $193 \mathrm{~L} 5 \mathrm{~s} 10 \mathrm{~d}$ & 63 & $128 \mathrm{~L} 12 \mathrm{~s} 8 \mathrm{~d}$ & & \\
\hline \multicolumn{7}{|c|}{ EPREUVE } \\
\hline ARGENT & & $221 \mathrm{~L}$ & & $215 \mathrm{~L}$ & \multirow{4}{*}{\multicolumn{2}{|c|}{ Aucune mention }} \\
\hline JETONS de 24 au marc & 402 & $820 \mathrm{~L} 16 \mathrm{~s} 4 \mathrm{~d}$ & 262 & $534 \mathrm{~L} 19 \mathrm{~s} 2 \mathrm{~d}$ & & \\
\hline BOUGIES (6 à la livre) & $220+x$ & \begin{tabular}{|c|}
$?$ \\
\end{tabular} & $220+x$ & $?$ & & \\
\hline BOUQUETS & 12 & $6 \mathrm{~L}$ & 12 & $6 \mathrm{~L}$ & & \\
\hline \multicolumn{7}{|c|}{ RECEPTION } \\
\hline ARGENT & \multirow{3}{*}{\multicolumn{2}{|c|}{ Aucune mention }} & \multirow{3}{*}{\multicolumn{2}{|c|}{ Aucune mention }} & & $146 \mathrm{~L}$ \\
\hline BOUGIES (6 à la livre) & & & & & 24 & $?$ \\
\hline \begin{tabular}{|l|} 
JETONS de 24 au marc \\
\end{tabular} & & & & & 84 & $171 \mathrm{~L} 10 \mathrm{~s} 3 \mathrm{~d}$ \\
\hline \begin{tabular}{|c|} 
TOTAL d'une \\
incorporation en 1767 \\
\end{tabular} & \multicolumn{2}{|c|}{$\begin{array}{c}1362 \text { L } 2 \text { s } 2 \text { d + 220+x bougies } \\
\text { (des } 12 \text { à la livre) }\end{array}$} & \multicolumn{2}{|c|}{$\begin{array}{l}1008 \text { L } 11 \text { s } 10 \mathrm{~d}+220+\mathrm{x} \\
\text { bougies (des } 12 \text { à la livre) }\end{array}$} & \multicolumn{2}{|c|}{$\begin{array}{c}317 \text { L } 10 \text { s } 3 \text { d + } 24 \text { bougies (des } \\
12 \text { à la livre) }\end{array}$} \\
\hline
\end{tabular}

Figure 6. Les droits fixés par l'arrêt de la Cour des monnaies du 23 mars 1767

\section{Détails des frais de réception d'une tailleresse en 1767 :}

* 1001 1. à remettre au prévôt des ajusteurs et tailleresses pour être employées « aux besoins et affaires de la Compagnie, sans qu'il puisse en être fait aucun partage »

* 42 1. pour l'arrêt de la Cour qui renvoie la tailleresse prêter serment devant les prévôts et lieutenants

$* 4$ 1. aux 2 clercs

* 4 bougies par officier de la Compagnie

* Des jetons d'argent de 24 au marc frappés sur les carrés de la Compagnie et non d'autres, distribués comme suit :

- $2 /$ officier

- $1 /$ monnayeur, $1 /$ ajusteur et $1 /$ tailleresse

L'arrêt de 1767 précise un point qui n'avait pas été abordé dans les deux textes précédents : si l'atelier reçoit des demandes d'accueillements de personnes, hommes ou femmes, pourvues de lettres d'interruption ${ }^{52}$, celles-ci devront payer des frais supplémentaires. En effet, leur parent, père ou mère, ne s'étant pas fait accueillir, cela représente une perte financière pour la Compagnie. Le nouvel accueilli, muni de ses lettres d'interruption, doit donc régler une partie des frais d'accueillement que son parent n'a pas payés. Cependant, la formulation utilisée dans l'arrêt n'est pas limpide: «Arrivant qu'il se présentât un sujet par lettres d'interruption, pour être reçu au droit de monnayeur, ajusteur \& tailleresse, parvenant à être agréé par la Compagnie, payera pour tout, à chaque monnayeur, ajusteur et tailleresse demi droit pour le défunt, en sus des droits ci-dessus \& des autres parts détaillées. » Le nouvel arrivant doit-il régler ses propres droits ainsi que la moitié des droits de son parent non accueilli, et donc donner «à chaque monnayeur, ajusteur \& tailleresse » la moitié des droits qu'il ou elle aurait dû recevoir, ou doitil régler ses propres droits à la Compagnie et donner à chaque monnayeur, à chaque ajusteur et à chaque tailleresse la moitié des droits totaux de son parent, ce qui semblerait absolument dissuasif? Les archives contiennent de nombreux cas de personnes accueillies alors qu'elles

52. Lettres délivrées par le roi permettant à un demandeur de se faire accueillir alors qu'il n'est pas fils ou fille d'anciens monnayeurs, ajusteurs ou tailleresses mais seulement petit-fils, petite-fille ou arrière-petit-fils, arrièrepetite-fille. 
n'avaient ni père ni mère à l'atelier ${ }^{53}$ et il serait étonnant qu'autant de prétendants et prétendantes aient accepté de payer si cher leur place à la Monnaie. La seconde interprétation du texte semble donc peu convaincante.

Au vu de ces tarifs, il est flagrant que les frais d'incorporation à la Compagnie entre 1690 et 1767 ont fortement augmenté, notamment pour les tailleresses (fig. 8).

\begin{tabular}{|c|c|c|c|}
\hline & $\mathbf{1 6 9 0}$ & $\mathbf{1 7 5 6}$ & $\mathbf{1 7 6 7}$ \\
\hline Argent & $100 \mathrm{l}$. & $1431.10 \mathrm{~s}$ & $146 \mathrm{l}$ \\
\hline Jetons & $981.8 \mathrm{~s}$ & $1761.17 \mathrm{~s} 10 \mathrm{~d}$ & $1711.10 \mathrm{~s} 3 \mathrm{~d}$ \\
\hline Total & $1981.14 \mathrm{~s}$ & $3191.7 \mathrm{~s} 10 \mathrm{~d}$ & $3171.10 \mathrm{~s} \mathrm{3} \mathrm{d}$ \\
\hline
\end{tabular}

Figure 7. Les frais de réception supportés par une nouvelle tailleresse à la Monnaie de Paris (hors les bougies)

Si l'on se concentre sur le cas particulier des tailleresses, on remarque que les frais supportés par elles ont presque doublé en un peu moins d'un siècle, alors que ceux des monnayeurs et des ajusteurs ont augmenté respectivement de $177 \%$ et $110 \%$ environ. Or, la livre ayant perdu, sur cette période, $50 \%$ de sa valeur du fait des nombreuses manipulations monétaires, et les prix à la consommation ayant par ailleurs doublé, la Cour des monnaies a probablement tenté de pallier à cette dévaluation par la hausse significative des droits de chaque nouvel(le) accueilli(e), notamment par l'augmentation du nombre des jetons d'argent à offrir aux travailleurs en poste. Cependant, les droits réglés par les nouveaux monnayeurs et les nouveaux ajusteurs ont connu une hausse bien plus importante que ceux des tailleresses, ce qui signifie que la «charge » de tailleresse a en quelque sorte perdu de sa valeur, puisque celle-ci n'a fait « que » suivre l'inflation due à la monnaie. On pourrait voir ici un signe de la perte d'importance et de reconnaissance de ce «métier » au cours du XVIII ${ }^{\mathrm{e}}$ siècle ainsi que l'illustration de l'intérêt de plus en plus limité porté par les contemporains à ce groupe de travailleuses.

Plus généralement, la situation des femmes dans le cadre privé et professionnel se dégrade au cours du XVIII ${ }^{\mathrm{e}}$ siècle français, annonçant le très misogyne XIX ${ }^{\mathrm{e}}$ siècle. Attention cependant à ne pas considérer la fin de l'Ancien Régime comme une période bannissant le travail féminin, puisque l'historiographie renouvelée de ces dernières années insiste clairement sur la faible « visibilité » des femmes, non sur leur « inactivité » ou leur cantonnement au foyer $\operatorname{clos}^{54}$.

\section{L'apprentissage en question}

La pratique de l'apprentissage, dont les tailleresses auraient apparemment été privées ${ }^{55}$, permet de revenir sur la place qu'elles tenaient dans le processus de production monétaire après l'introduction du coupoir. Un registre de la Monnaie de Paris vient en effet contredire l'affirmation des juristes contemporains quant à un apprentissage des femmes ${ }^{56}$. Celui-ci compile tous les accueillements d'hommes et de femmes à la Monnaie de Paris entre 1587 et 1662, dans lequel se trouvent 11 mentions d'épreuve pour les nouvelles tailleresses accueillies entre 1641 et 1651 , sauf une :

53. Elles faisaient en général valoir l'accueillement d'un grand-père ou d'une grand-mère à la Monnaie de Paris au lieu de celui de leur père ou de leur mère pour faire justifier leur demande d'incorporation, comme Marie Cécile et Marie Claude Langlois, accueillies le 3 juillet 1690 (AN, $\mathrm{Z}^{1 \mathrm{~B}}$ 572).

54. Entre autres DUBY, PERROT 1991, TRUAND 1996, CROWSTON 2000, BeAuvalet-BoutouYRIE 2003, GODINEAU 2003, CROWSTON 2008.

55. АвOT DE BAZINGHEN 1764, p. 307.

56. CAEF, MP, msf 45. 
«- 16 avril 1641. Réception de Marie de Compans, femme de Guillaume Boucher, greffier en chef de la Cour des Aydes, tailleresse, laquelle a fais son épreuve en présence des prévots, lieutenants, ouvriers et monnoyers.

- Du même jour. Réception de Marguerite de Compans, femme Lescot (marchand bourgeois de Paris), tailleresse, avec pareille épreuve.

- $1^{\text {er }}$ février 1642. Réception de Gillette Pijart, femme Boulour, tailleresse, avec épreuve comme ci-dessus.

- Du même jour. Réception de Geneviève Pijart, femme Trousseuille, tailleresse, avec pareille épreuve.

- 5 avril 1644. Réception de Marguerite Pijart, femme Choisin, et de Françoise Pijart, femme Pieques, tailleresse, avec pareilles épreuves comme ci-dessus.

- 3 décembre 1648. Réception de Renée Le Tellier, veuve Bastier, tailleresse, avec épreuve comme ci-dessus.

- 7 mars 1651. Réception d'Anne Auboust sans épreuve.

- 5 septembre 1651. Réception de Marie Grandcerf, tailleresse, avec pareille épreuve.

- Du même jour. Réception de Marie Agnès Auboust, tailleresse, avec pareille épreuve.

- Du même jour. Réception de Martine Auboust, tailleresse, avec pareille épreuve.

- 5 décembre 1651. Réception de Marguerite Rolland, tailleresse, avec pareille épreuve

- Du même jour. Réception de Nicole Auboust, tailleresse, avec pareille épreuve. »

Il précise même, pour l'une d'entre elle, le 16 avril 1641 :

« Damoiselle Marie de Compans, femme de noble homme M. Guillaume Boucher, conseiller secrétaire du roy et huissier en chef de sa Court des Aydes (...) à esté receue et acueillie du costé des ouvriers et tailleresses de ladite Monnoye faict et presté le serment en tel cas requis et acoustumé et a l'instant faict espreuve en l'une des fournaizes de ladite Monnoye en présences de messieurs les prévostz lieutenant, ouvrier et monnoyeurs y assistants. »

L'hypothèse la plus probable quant à la raison de cette vague momentanée d'apprentissage, et donc d'épreuves imposées aux nouvelles venues à l'atelier, est que dans les années 1640 sont introduits définitivement les moulins, balanciers et surtout les coupoirs à la Monnaie de Paris, à l'occasion de la création des louis d'or et des écus d'argent ${ }^{57}$. Or jusqu'à présent les tailleresses travaillaient à la main - tout comme les ajusteurs et les monnayeurs. Puisque le coupoir réalisait désormais leur travail, l'administration monétaire les reclassa en leur confiant une autre tâche manuelle, la même que celle réalisée par les ouvriers ajusteurs : le limage à l'aide d'écouennes. Elles durent donc être formées à ce geste. Cependant, à l'inverse des femmes, les hommes continuèrent à réaliser leur épreuve jusqu'à la fin de l'Ancien Régime. Il est difficile d'expliquer pourquoi la Cour des monnaies sembla n'imposer une formation qu'à une seule génération de tailleresses. Devaient-elles travailler seules et transmettre leur savoir elles-mêmes aux futures ouvrières, signe d'une première forme d'exclusion ? Futures ouvrières qui suivraient alors une sorte d'apprentissage non reconnu auprès de leurs ainées. Il est également possible que, puisque les tailleresses ne devaient recevoir que le quart des matières à ajuster ${ }^{58}$ - et les ajusteurs les trois autres quarts -, leur formation soit passée au second plan. Cela serait une aberration en matière de productivité, mais un calcul réfléchi si l'on souhaitait les exclure, à terme, de l'atelier.

57. Déclaration du 31 mars 1640 donnée à Saint-Germain-en-Laye pour l'or et édit de septembre 1641 donné à Péronne pour l'argent.

58. ABOT DE BAZINGHEN 1764, t. 2, p. 303. Cette division du travail est semble-t-il une persistance des usages du Moyen Age, qui voulait que les effectifs de tailleresses ne dépassent pas un quart à un cinquième des effectifs totaux des officiers de fabrication. 


\section{Un conflit du travail confirme l'implication des tailleresses aux travaux d'ajustage}

L'année 1662 offre paradoxalement un bel exemple de la résistance dont pouvaient faire preuve les tailleresses face à une majorité masculine de travailleurs désireux de les pousser hors de l'établissement. Un registre de la Monnaie de Paris rend compte d'un conflit ${ }^{59}$, opposant les « ouvriers » ajusteurs aux tailleresses de l'atelier, portant sur les quantités de matière à ajuster par chaque groupe et, surtout, des revenus à en tirer. Neuf arrêts de la Cour des monnaies, rendus entre le 28 mars et le 9 août 1662, relatent les affrontements des deux parties. Le premier arrêt expose la requête des tailleresses « servant actuellement au travail de la monnoye de Paris » adressée à la Cour le 23 mars. Ces dernières se plaignent que, depuis l'établissement officiel du moulin ${ }^{60}$, les ouvriers et leur prévôt François Lefevre ne leur reversent plus les droits sur les brèves qui doivent normalement leur revenir. Depuis l'établissement du moulin les ajusteurs devaient, nous l'avons dit, laisser un quart des flans à ajuster aux tailleresses. La Cour leur répond favorablement, oblige les ouvriers à leur donner ce qui leur revient et leur demande de cesser de les importuner dans leur travail. La suite des textes n'est qu'une longue succession de requêtes déposées soit par les tailleresses - demandant la restitutions de leurs droits - soit par le prévôt, le lieutenant et les ouvriers - s'opposant à la restitution des dites sommes sans jamais se justifier.

L'intérêt de ce corpus de textes réside dans la capacité du groupe minoritaire des officiers de fabrications des monnaies à faire entendre sa voix, alors qu'il ne dispose pas de représentant

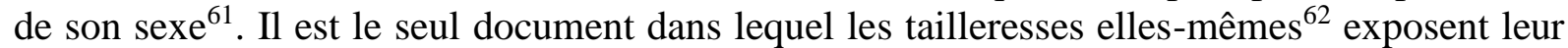
implication dans le processus d'ajustage et argumentent sur le bien-fondé de l'action qu'elles mènent contre les ajusteurs. La grande majorité des arrêts de la Cour des monnaies fait suite aux requêtes déposées par les tailleresses, ce qui révèle plusieurs choses: d'abord que l'atmosphère au sein de la Monnaie entre ouvriers et tailleresses n'était déjà au beau fixe en 1662 et que les ajusteurs avaient, semble-t-il, beaucoup de mal à accepter le reclassement des tailleresses qui venaient en quelque sorte «voler» leur travail, tout comme le coupoir avait empiété sur celui des femmes. Ensuite, que malgré le développement de ce climat tendu, empreint d'une certaine misogynie, la Cour maintenait intacts les droits des tailleresses à la représentation et aux contestations qu'elles pouvaient formuler à l'encontre de leurs homologues masculins trop peu partageurs. Les neufs arrêts nous apprennent plusieurs éléments dans le détail :

- C'est à partir du 23 mars 1662 que le prévôt des ouvriers a cessé de leur reverser leurs droits. Nous n'en connaissons pas la raison.

- La Cour des monnaies a plusieurs fois condamné les ouvriers et notamment leur prévôt Lefevre à restituer les sommes dues aux tailleresses, sans qu'ils obtempèrent. Ces sommes ont été calculées par Jacques Bourgeois, ancien garde de la Monnaie de Paris mandaté par la Cour des monnaies, à qui le prévôt a dû confier les registres du bureau de la Monnaie afin qu'il puisse calculer précisément ce que représentait le quart des matières ajustées. Ainsi la Cour était-elle en capacité de fixer précisément la somme que les ouvriers devaient reverser aux tailleresses pour le travail qu'elles avaient réalisé entre le 4 avril et le 17 mai $1662^{63}$, soit $2371.16 \mathrm{~s}$. Nous

59. CAEF, MP, msf $4^{\circ} 81$.

60. Édit de mars 1645 enregistré en décembre 1645. CONSTANS 1685, p. 321, preuves p. 235.

61. En effet, les tailleresses et les ajusteurs formaient une « sous-compagnie » représentée et dirigée par un prévôt et un lieutenant des ajusteurs qui ne pouvait être une femme.

62. Par l'intermédiaire de leur procureur Boyer. Les ajusteurs étaient eux représentés par le procureur Mussot.

63. Nous ne savons pas pourquoi la période commence le 4 avril, date de l'arrêt de la Cour qui ordonne que le quart des droits à reverser aux tailleresses soit calculé, et non le 23 mars, date du dépôt à la Cour par les tailleresses de leur première requête. 
savons par ailleurs qu'en 1664 les tailleresses étaient 46 à l'atelier ${ }^{64}$ : le gain est donc d'un peu plus de 5 1. pour chacune. Les ouvriers étaient quant à eux au nombre de $24^{65}$. Si $2371.16 \mathrm{~s}$ représentaient le quart des profits réalisés sur l'ajustage entre le 4 avril et le 17 mai 1662, on considère que la totalité des droits sur les brèves s'élevait à 950 l. environ et que les trois-quarts représentaient un peu plus de 7101 . Cela correspond à un gain théorique d'un peu moins de 30 1. par ouvrier pour une période d'activité d'un mois et demi. Refuser les 51 . à chacune des tailleresses leur aurait donc permis de gagner environ 101 . de plus chacun.

- Les ouvriers se sont opposés aux ordres successifs de la Cour des monnaies de rémunérer les tailleresses en promulguant un texte en interne, cassant les rappels à la loi et refusant de restituer les sommes dues aux tailleresses. Ce texte interne fut à son tour cassé et annulé par la Cour qui menaça les ouvriers de 3000 livres d'amende en cas de récidive. Les sommes revenant aux tailleresses furent finalement saisies par la Cour des monnaies elle-même.

- Maître François Lefevre, prévôt des ouvriers, son lieutenant et les ouvriers devaient désormais livrer aux tailleresses le quart de tout l'ouvrage à ajuster au bureau de la Monnaie et non en la chambre de l'ajustage comme cela se faisait jusqu'à présent. Celles-ci obtiennent une chambre particulière pour l'or, spécialement dédiée aux femmes, pour ne plus être importunées dans leur travail. L'existence de cette chambre est encore attestée plus d'un siècle plus tard par $l^{\prime}$ « inventaire du monoyage et de l'ajustage pour tous les ustenciles le 28 février $1782^{66}$ » où l'on parle, en plus d'une chambre de l' « ajustage des tailleresses à l'argent », de la « chambre à or des dames ». Cet inventaire permet par ailleurs de constater que les femmes bénéficiaient exactement du même matériel que les hommes : tables d'ajustage, balances posées sur des tables à tiroirs fermant à clefs pour peser les espèces, grands chandeliers, tables pour les vérificateurs, poids, armoires, dénéraux, tabourets et coffres forts.

\section{Évaluation de la productivité des ajusteurs et des tailleresses}

Il est difficile d'évaluer ce que pouvait être la productivité des officiers de fabrication des monnaies. Un mémoire des monnayeurs et ajusteurs ${ }^{67}$ - qui ne mentionne cependant jamais les tailleresses comme se joignant aux revendications - nous y aide en rendant compte du travail réalisé par les ajusteurs en une journée :

«L'on supose qu'il est apporté le lundy 300 marcs d'or, le directeur les fait aussitôt mettre en fonte et fait mander les ajusteurs pour le mardy six heures du matin, lesquels toutes affaires cessantes se trouvent au nombre de 25 (dont 20 prennent les limes et les 5 autres conduisent). Les 20 destinés à ajuster prennent chacun 15 marcs et rendent le tout sur les 11 heures, scavoir environ 200 marcs d'ajustés et environ 100 marcs en rebut et limaille. »

Le mémoire poursuit en exposant les pertes de temps et d'argent considérables imposées et facturées aux ajusteurs, consécutives aux nombreuses refontes des rebuts et limailles, refontes exigées par le directeur de la Monnaie pour ne pas perdre d'argent. L'information la plus importante est la quantité de marcs que pouvait ajuster un ajusteur, et donc doute une tailleresse, en un temps donné. Un ouvrier ajusteur mettait 5 heures pour ajuster 15 marcs d'or dans les années 1770-1780, ce qui correspond à une productivité de 3 marcs ajustés par heure travaillée, mais on doit prendre en compte la tendance à forcer le trait quand il s'agit de faire remonter aux autorités quelques revendications. Cela représente l'ajustage d'un peu plus de cent louis par

64. AN, T $1491^{12}$.

65. Ne sont pas comptés ici les 14 recuiteurs que comptait l'atelier cette année-là.

66. AN, T149126.

67. Idem, mémoire rédigé entre 1771 et 1789. 
heure ${ }^{68}$, soit un louis toutes les 30 secondes environ. Cette productivité masculine pourrait correspondre à celle des femmes qui effectuaient les mêmes gestes, mais dans la chambre qui leur était réservée depuis $1662 \ldots$

\section{De l'intérêt à devenir tailleresse}

Il est délicat d'évaluer le retour sur investissement espéré par les officiers particuliers des monnaies déboursant des frais d'accueillement si élevés pour pouvoir entrer à la Monnaie. Le cas des tailleresses en particulier soulève des interrogations, car à l'inverse des lingères, des couturières, des mercières ou les dentelières, elles n'exerçaient à priori pas un métier leur offrant une indépendance financière. Où se trouvait donc l'intérêt d'entrer à la Monnaie ? La procédure à suivre pour mener une telle étude est théoriquement assez bien balisée.

Il est d'abord nécessaire de déterminer les privilèges et exemptions dont jouissaient les officiers des Monnaies, régulièrement rappelés dans la législation royale. Ces privilèges ont été originellement octroyés par Philippe le Bel en 1296 :

«Exemption de toute tailles, taillons, gabelles, impositions, subventions, coûtumes et tous droits de voirie, quatrième, huitième, treizième, vingtième, cinquantième, centième, chauffées, subsides, aydes, chevauchées, logemens et entretien de gens de guerre, charges d'églises et d'hôpitaux, solde de cinquante mille hommes de guerre, levées ordinaires et extraordinaires, ponts, portes, péages, passages, garde des portes, guet, sentinelle, fortification, entrée des villes, réparations, tutelles, curatelles, établissement de commissaires par justice, coûtumes de vin et bière, soit grande ou petite, posées ou à poser, soit qu'il fut de leur propre crû ou voie d'achat, avec cette prérogative de ne pouvoir être appelés devant d'autres juges que les généraux provinciaux des monnaies. ${ }^{69}{ }^{»}$

Mais des lettres patentes de Louis XVI, données à la Muette en octobre 1782, donnent une précision quant à la jouissance des exemptions d'octroi pour les monnayeurs, ajusteurs et tailleresses de la Monnaie de Paris $^{70}$ :

«Article V - L'exemption des entrées en droits d'octrois confirmée par l'article III à nos officiers, monnoyeurs, ajusteurs et tailleresses, sur les vins, bière, cidre et eau de vie de leur consommation, ne sera point étendue à nos officiers et monnoyeurs de la Monnoie de Paris, et ils continueront d'être assujettis auxdits droits comme par le passé. »

Cette limite non négligeable vient semer le trouble dans les connaissances jusqu'ici compilées sur les droits, privilèges et exemptions dont jouissaient les officiers des Monnaies. En province, les monnayeurs, ajusteurs et tailleresses profitent des exemptions d'octroi, ce qui participe, avec l'exemption de taille, à l'attractivité du poste qu'ils convoitent, comme le démontre notamment Jérôme Jambu pour la Monnaies de Caen ${ }^{71}$. Il semblerait donc, à la vue de ces deux textes presque contradictoires, qu'il y ait eu une modification des privilèges et exemptions octroyés aux officiers particuliers parisiens entre 1296 et 1782. Un document conservé dans les archives de la Compagnie des monnayeurs de Paris datant de $1604^{72}$ vient alimenter cette hypothèse d'une modification des privilèges au fil des siècles :

68. Le louis dont la fabrication fut ordonnée par l'édit de janvier 1726, demeurait au titre de 22 carats mais était taillé à 30 au marc, alors que celui de 1640 l'était à 36,25 au marc (CHARLET 1996, p. 71).

69. CAEF, MP, msf $8^{\circ} 4526$, édit de janvier 1719 réglementant les privilèges des monnayeurs, ajusteurs et tailleresses des Monnaies. La citation est ici le rappel des privilèges originellement octroyés par Philippe le Bel à ses monnayeurs, ajusteurs et tailleresses quand il les dispersa et les sédentarisa dans plusieurs Monnaies du royaume en 1296, dont celle de Paris.

70. Paris, imprimerie royale, 1783.

71. JAMBU 2013, p. 173-177.

72. AN, T $1491^{9}$. 
« Du $2^{\mathrm{e}}$ avril 1604.

Sentence des esleus de Paris portant damoiselle Marie Voulier, tailleresse en la monoye de Paris, estre franc, quicte et exempte des droicts d'entrée des cinq sols antiers par muid de vin et des 4 sols 2 deniers et dix deniers aussy pour muid de vin. »

Ces quelques lignes prouvent qu'en 1604 au moins, les tailleresses, et certainement avec elles le reste des officiers particuliers de la Monnaie de Paris, étaient encore exemptées de l'octroi sur le vin. La question du « retour sur investissement»d'une incorporation à la Monnaie est donc difficilement quantifiable en ce qui concerne les exemptions d'octroi, tant la législation reste à éclaircir.

L'étape suivante serait de parvenir à évaluer les gains que pouvaient représenter les exemptions de taxes pures et d'impôts, ordinaires et extraordinaires, dixième, vingtième, etc. Il est par exemple possible de déterminer les gains que représentait l'exemption de taille grâce aux rôles conservés. Cependant attention, tous les officiers particuliers de la Monnaie ne jouissaient pas de ce privilège, la Cour des Aides imposant régulièrement l'établissement d'un numerus clausus d'exemptés de taille et de taillons afin de ne pas surcharger les contribuables. L'exemption du logement et de l'entretien de soldats devait représenter, quant à elle, un réel intérêt financier, tant ces «gens de guerre» constituaient une lourde charge quand ils s'installaient chez les particuliers. Enfin, il faudrait quantifier ce que représentaient financièrement des exemptions telles que «ponts, portes, péages, passages (...), fortification, réparations », charges sans doute plus ponctuelles que régulières.

Si la Cour des Aides restreignait le nombre des officiers particuliers des monnaies exemptés de taille, si ces derniers n'étaient pas exemptés d'octroi et sachant que les revenus issus du monnayage et de l'ajustage étaient modiques ${ }^{73}$, sans doute le retour sur investissement n'étaitil pas, à Paris, d'ordre financier. C'est du côté de la jouissance de privilèges honorifiques (droit de porter l'épée par exemple), judiciaires (dispenses de tutelles et de curatelles, soumission à des juges particuliers différents des autres justiciables), des exemptions militaires («garde des portes, guet, sentinelle »), bien que les femmes n'y fussent pas soumises, mais surtout, pour elles, du côté de la protection offerte par la Compagnie, sorte d' " assurance vieillesse, maladie et accidents du travail » assurée par sa charité, la Maladrerie du Roule, dotée d'un «pot commun » à destination de ceux qui en avaient besoin, qu'il faut regarder.

\section{Conclusion}

L'introduction du progrès technique à de la Monnaie de Paris au milieu du XVII ${ }^{\mathrm{e}}$ aurait pu avoir plusieurs conséquences, notamment celle de réorganiser l'attribution les tâches de chacun dans le processus de fabrication monétaire. Or tel ne fut pas le cas, sauf a minima pour les tailleresses qui se virent associées aux ajusteurs, réalisant finalement le même geste sur le flan sortant du coupoir qu'elles ne maniaient pas. Il aurait cependant été concevable de leur en confier l'utilisation. Ce ne fut pas le cas.

En outre, ces femmes n'ont pas pu se constituer en groupe d'ouvrières à part entière. Au lieu de cela elles furent «déclassées », passant du statut d' « ouvrières officiers » à part entière à celui de «sous officier», main d'œuvre d'appoint pour des ajusteurs trop souvent réticents à leur verser leurs droits sur les brèves qu'elles avaient elles-mêmes travaillées. Elles étaient, par exclusion, les éternelles absentes des temps forts de la vie de la Monnaie, élections des représentants, des messes, des célébrations, des banquets, etc.

73. Ces droits étaient d'un sol par marc d'argent et de deux sols par marc d'or monnayé. Ils étaient assez régulièrement complétés de gratifications extraordinaires indexées sur les productions. 
Malgré tout, il serait erroné de conclure que, dès le XVII ${ }^{\mathrm{e}}$ siècle, les tailleresses de la Monnaie de Paris furent poussées hors de l'atelier, puisqu'un conflit tel celui de 1662 prouve qu'elles ont résisté à cet environnement détérioré, avec l'appui, plus ou moins convaincu, de la Cour des monnaies de Paris. Il semble qu'en réalité, le statut de ces femmes ait commencé à changer à partir de l'introduction des machines et moulins mais que l'évolution fut lente et n'aboutit nullement à leur disparition. Les vagues successives de réceptions lors des périodes de forte production monétaire en témoignent encore.

La conclusion la plus juste de cette étude est probablement celle d'une perte d'importance, de considération et de prestige des tailleresses par rapport aux monnayeurs et aux ajusteurs, d'avantage fruit des comportements des hommes de l'atelier que de l'administration monétaire, mais certainement pas celle de leur disparition de l'atelier monétaire parisien moderne ${ }^{74}$.

\section{Bibliographie}

ABAD 2002 : R. ABAD, Le grand marché : l'approvisionnement alimentaire de Paris sous l'Ancien Régime, Paris, 2002.

Авот De BAZINGHEN 1764 : Fr. АвOT DE BAZINGHEN, Traité des monnoies et de la jurisdiction de la Cour des monnoies, en forme de dictionnaire, I, Paris 1764.

AUBREE 1903 : E. AUBREE, Une famille de monnayeurs rennais aux XVI ${ }^{e}$, XVII et XVIII siècles. Étude sur la monnaie de Rennes, Rennes, 1903.

BeAuVALET-BOUTOUYRIE 2003 : S. BEAUVALET-BOUTOUYRIE, Les femmes à l'époque moderne (XVI ${ }^{e}$ XVIII ${ }^{e}$ siècles), Paris, 2003.

BoIZARD 1692 ET 1714 : J. BoIZARD, Traité des monoyes, 2 vol., Paris, 1692 ( $2^{\mathrm{e} e ́ d .) ~ e t ~} 1714$ (?e éd.).

Blanchet, Dieudonne 1912/1936: A. Blanchet et A. Dieudonne, Manuel de numismatique française, 4 vol., Paris, 1912-1936.

CHARLET 1996 : Chr. CHARLET, Monnaies des rois de France, de Louis XIII à Louis XVI, Paris, 1996.

CLAIRAND 1996 : A. CLAIRAND, Monnaies de Louis XV, le temps de la stabilité monétaire, 1726-1774, Paris, 1996.

ClairAnD 2014a : A. ClairAnd, Le collège des monnayeurs, ajusteurs et tailleresses de la Monnaie de Lille (1686-1790), Revue du Nord, 2014/3, à paraître.

ClAIRAND 2014b : A. ClAIRAND, Le personnel de l'atelier monétaire de Lille (1685-1790), mémoire de master 1, Université Charles de Gaulle-Lille 3, 2014.

CONSTANS 1658 : G. CONSTANS, Traité de la Cour des Monnoyes et de l'estendue de sa jurisdiction, Paris, 1658.

CROWSTON 2000 : C. CROWSTON, "Engendering the Guilds : Seamstresses, Tailors and the Clash of Corporate Identities in Old Regime France", French historical studies, 23/2, Duke University Press, 2000.

Crowston 2008 : C. CROWSTON, "Women, Gender, and Guilds in Early Modern Europe: An Overview of Recent Research", IRSH, 2008, p.19-44.

DARNIS 1988 : J.-M. DARNIS, La Monnaie de Paris. Sa création et son histoire du Consulat et de l'Empire à la Restauration (1795-1826), Abbeville, 1988.

D’AVENEL 1894-1926 : G. D’AVENEL, Histoire économique de la propriété, des salaires, des denrées et de tous les prix en général, depuis l'an 1200 jusqu'en l'an 1800, Paris, 1894-1926.

DelCAMP 2014 : M. DelCAMP, Les tailleresses de l'atelier monétaire de Paris, 1645-1789, mémoire de master 2, Université Paris 1 Panthéon-Sorbonne, 2014.

DIDEROT, d'AlEMBERT 1772 : D. DIDEROT et F. d'ALEMBERT, Encyclopédie ou Dictionnaire raisonné des sciences, des arts et des métiers, Genève, 1772 ( $2^{\mathrm{e}}$ éd.) ; planches, Paris, 1759 et suiv.

Duby, Perrot 1991 : G. Duby et M. PERrot (dir.), Histoire des femmes en Occident. III, XVI ${ }^{e}$-XVIII siècle, Paris, 1991.

74. Nous tenons à remercier sincèrement les personnes qui ont rendu possible la rédaction de cet article et qui n'ont jamais hésité à dispenser leurs précieux conseils, MM. Jérôme Jambu, Christian Charlet et Arnaud Clairand. 
DUPRE DE SAINT-MAUR 1746 : N. F. DUPRE DE SAINT-MAUR, Essai sur les monnoies, ou réflexions sur le rapport entre l'argent et les denrées, Paris, 1746.

DURAND 1996 : Y. DURAND, Les fermiers généraux au XVIII siècle, Paris, 1996.

GODINEAU 2003 : D. GODINEAU, Les femmes dans la société française. $16^{e}-18^{e}$ siècle, Paris, 2003.

JAMBU 2013 : J. JAMBU, Tant d'or que d'argent. La Monnaie en Basse-Normandie à l'époque moderne (XVI ${ }^{e}$-XVIII ${ }^{e}$ siècles), Rennes, 2013.

LACHIVER 1982 : M. LACHIVER, Vin, vigne et vignerons en région parisienne du XVII ${ }^{e}$ au XIX ${ }^{e}$ siècle, Pontoise, 1982.

MAZEROLle 1907 : F. MAZEROLLe, La Monnaie. Les bâtiments, les ateliers, le musée, Paris, 1907.

PANCKOUCKE 1784-1788 : Encyclopédie méthodique, arts et métiers mécaniques, Paris, 1784-1788 (réédition de l'article «L'Art du monnayage », Arras, 1981).

SAINT-JUliEn (DE) et BIENAYME 1886 : A. DE SAINT-JULIEN et G. BIENAYME, Les droits d'entrée et d'octroi à Paris depuis le douzième siècle, Paris, 1886.

SALAÜN 2003 : G. SALAÜN (dir.), L'atelier monétaire de Nantes sous l'Ancien Régime, Nantes, 2003.

SPOONER 1956 : F. SPOONER, L'économie mondiale et les frappes monétaires en France, Paris, 1956.

THUILliER 2005 : G. THUILliER, La Réforme monétaire de 1785 : Calonne et la refonte des louis, Paris, 2005.

TRUAND 1996 : C. TRUAND, La maîtrise d'une identité ? Corporations féminines à Paris aux XVI et XVIII ${ }^{\mathrm{e}}$ siècles, CLIO. Histoire femmes et sociétés, ${ }^{\circ} 3,1996$, p. ? 